\title{
The Mass and Size of Photons in the 5-Dimensional Extended Space Model
}

\author{
V. A. Andreev1, D. Yu. Tsipenyuk ${ }^{2 *}$ \\ ${ }^{1}$ Lebedev Physical Institute of RAS, Moscow, Russia \\ ${ }^{2}$ Prokhorov General Physics Institute of RAS, Moscow, Russia \\ Email: andrvlad@yandex.ru, 'tsip@kapella.gpi.ru
}

Received 29 May 2016; accepted 11 July 2016; published 14 July 2016

Copyright (C) 2016 by authors and Scientific Research Publishing Inc.

This work is licensed under the Creative Commons Attribution International License (CC BY). http://creativecommons.org/licenses/by/4.0/

(c) (i) Open Access

\section{Abstract}

We propose the generalization of Einstein's special theory of relativity (STR). In our model, we use the $(1+4)$-dimensional space $G$, which is the extension of the $(1+3)$-dimensional Minkowski space $M$. As a fifth additional coordinate, the interval $S$ is used. This value is constant under the usual Lorentz transformations in $M$, but it changes when the transformations in the extended space $G$ are used. We call this model the Extended space model (ESM). From a physical point of view, our expansion means that processes in which the rest mass of the particles changes are acceptable now. In the ESM, gravity and electromagnetism are combined in one field. In the ESM, a photon can have a nonzero mass and this mass can be either positive or negative. It is also possible to establish in the frame of ESM connection between mass of a particle and its size.

\section{Keywords}

Photon, Mass, Size, 5-Dimensional Space, Extended Space Model, Gravitation, Special Theory of Relativity

\section{Introduction}

We consider the Extended space model (ESM), which is a generalization of Einstein's special theory of relativity (STR). The ESM is formulated in a 5-dimensional space, or more specifically in a (1 + 4)-dimensional space with the metric $(+----)$. Thus, we work in a space with coordinates $(t, x, y, z, s)$ and metric $(+----)$. The objects under consideration are located on a cone

$$
(c t)^{2}-x^{2}-y^{2}-z^{2}-s^{2}=0 .
$$

${ }^{*}$ Corresponding author. 
The foundations and different properties of this theory are presented in Ref. [1]-[6]. Here we briefly recall its main statements and describe the structure of the Extended space.

In the STR, the rest mass $m$ of a particle is a Lorentz scalar. For photons, $m=0$. The main idea of the ESM is that the mass $m$ is not a Lorentz scalar and can vary under external influences.

Such particle having a mass $m$, corresponds to a hyperboloid in Minkowski space, in the limiting case this hyperboloid degenerates into a cone.

$$
s^{2}=(c t)^{2}-x^{2}-y^{2}-z^{2}
$$

Since the change of the mass of a particle corresponds its transition from one hyperboloid to the other, i.e. change of the corresponding interval, it seems natural to choose interval $s$ as an additional fifth coordinate. Thus, we will work in a space with coordinates $(t, x, y, z, s)$ and metric $(+----)$. The objects under consideration are located on a cone

$$
(c t)^{2}-x^{2}-y^{2}-z^{2}-s^{2}=0 .
$$

We denote this space as $G(1,4)$. The Minkowski $M(1,3)$ space is a subspace of $G(1,4)$. An interval in the Minkowski $M(1,3)$ space plays a role of the fifth coordinate in the $G(1,4)$ space. We designate this coordinate by the letter $S$. The other coordinates are designated as $T, X, Y, Z$. One of the characteristic features of the ESM is that the particle's rest mass $m$ is $s$ variable quantity and a photon, moving in a medium with refraction index $n>1$, and a nonzero mass is acquired. This mass can be both positive and negative.

The usual $(1+2)$-dimensional cones and hyperboloids occur as sections of the surface (3) by hyperplanes $s=s_{0}$. In the space $G(1,4)$ one can constructed in usual way the objects that have different tensor nature and transform appropriately under linear transformations of the $G(1,4)$ space (Ref. [7]).

The 5-dimensional ESM has a number of advantages compared to STR. Firstly, it is a more symmetric theory. In this model, the energy, momentum and mass are equivalent and can be transformed into each other. Secondly, in this model there is no fundamental difference between massive and massless particles and they can be transformed into each other either. In addition, under the ESM electromagnetic and gravitational fields are combined into a single field. This field is investigated in papers [2] [5]. It is shown that with the help of rotations in $G(1,4)$ space one can transform the electromagnetic field into four other fields with gravitational properties.

In this work, we will show that it is possible to compare a nonzero mass to a system of photons, and that it is possible to establish connection between mass of a photon and its size.

The various aspects of the concept of “mass" in STR was discussed by Okun' [8]-[10].

\section{The Refractive Index in the Extended Space}

In Minkowski space $M(1,3)$ a 4-vector of energy and momentum

$$
\tilde{p}=\left(\frac{E}{c}, p_{x}, p_{y}, p_{z}\right)
$$

is associated to each particle (Ref. [11]). In the extended space of $G(1,4)$, we completes its to 5-vector

$$
\bar{p}=\left(\frac{E}{c}, p_{x}, p_{y}, p_{z}, m c\right) \text {. }
$$

For free particles, the components of the vector (5) satisfy the equation

$$
E^{2}=c^{2} p_{x}^{2}+c^{2} p_{y}^{2}+c^{2} p_{z}^{2}+m^{2} c^{4} .
$$

It is well-known relation of relativistic mechanics, which relates the energy, momentum and mass of a particle. Its geometric meaning is that the vector (5) is isotropic, i.e. its length in the space $G(1,4)$ is equal to zero. However, in contrary to the usual relativistic mechanics, we now suppose that the mass $m$ is also a variable, and it can vary at motion of a particle on the cones (3), (6). It should be understood so that the mass of the particle changes when it enters the region of the space that has a nonzero density of matter or field. Since in such areas the speed of light is reduced, they can be characterized by value $n$ - the refractive index of a medium. The parameter $n$ relates the speed of light in vacuum $c$ with the speed $v$ of light in a medium. 


$$
v=c / n \text {. }
$$

We compere parameter $n \geq 1$ to each form of medium or field. For example the refractive index of a gravitational field that is described by the Schwarzchild solution reads [9],

$$
n(r)=1+\frac{r_{g}}{r}=1+\frac{2 \gamma M}{r c^{2}} .
$$

Here $r_{g}<r$ is the gravitational radius and $M$ is a dot mass, which generates the Schwarzchild field.

A set of variables (5) forms a 5-pulse, its components are conserved, if the space $G(1,4)$ is invariant under the corresponding direction. In particular, its fifth component $p_{4}$, having sense of mass, does not change if the particle moves in the area with constant value $n$.

The gravitational effects in ESM were discussed in Ref. [3]-[5].

\section{The Vectors of the Free Particles}

In the usual relativistic mechanics and field theory the mass of a particle is constant, and for particles with zero masses and nonzero rest masses different methods of description are used. The particles with nonzero rest masses are characterized by their mass $m$ and speed $\vec{v}$. The particles with zero mass (photons) are characterized by frequency $\omega$ and wavelength $\lambda$. These $\omega$ and $\lambda$ are connected with energy $E$ and momentum $\vec{p}$ sa follows

$$
E=\hbar \omega, \quad \vec{p}=\frac{2 \pi \hbar}{\lambda} \vec{k}
$$

The 4-vector

$$
\tilde{p}_{m}=\left(\frac{E}{c}, \vec{p}\right)=\left(\frac{m c}{\sqrt{1-\beta^{2}}}, \frac{m \vec{v}}{\sqrt{1-\beta^{2}}}\right), \beta^{2}=\frac{v^{2}}{c^{2}}
$$

corresponds to a particles with nonzero rest mass.

The 4-vector

$$
\tilde{p}_{\omega}=\left(\frac{\hbar \omega}{c}, \frac{2 \pi \hbar}{\lambda} \vec{k}\right)=\left(\frac{\hbar \omega}{c}, \frac{\hbar \omega}{c} \vec{k}\right)
$$

corresponds to a particles with zero mass.

The length of the 4-vector $\tilde{l}=\left(x_{0}, x_{1}, x_{2}, x_{3}\right)$ is defined in accordance with the metric $(+---)$ of the Minkowski space $M(1,3)$ It equal to

$$
\tilde{l}^{2}=x_{0}^{2}-x_{1}^{2}-x_{2}^{2}-x_{3}^{2} .
$$

It follows from (12) that length of a massive vector (10) $\tilde{p}_{m}^{2}=m^{2} c^{2}$, therefor it is not an isotropic vector. And length of a photon vector (11) $\tilde{p}_{\omega}^{2}=0$, so it is an isotropic vector. It is the difference between massive and massless particles in the frame of STR.

In the frame of our approach, there is no difference between massive and massless particles, and therefore one can establish a connection between two methods of description of these two sorts of particles. This can be done using the relation (9) and the hypothesis of de Broglie, according to which these relations hold for the massive particles. Now, substituting (9) in (5), we obtain the relation between the mass $m$, frequency $\omega$ and wavelength $\lambda$

$$
\begin{aligned}
& \omega^{2}=\left(\frac{2 \pi c}{\lambda}\right)^{2}+\frac{m^{2} c^{4}}{\hbar^{2}} . \\
& \omega=\frac{m c^{2}}{\hbar \sqrt{1-\beta^{2}}}, \lambda=\frac{2 \pi \hbar}{m v} \sqrt{1-\beta^{2}} .
\end{aligned}
$$

It follows that if $v \rightarrow 0, \lambda \rightarrow \infty$, but $\omega \rightarrow \omega_{0} \neq 0$. Here $\omega_{0}$ determines the energy of a particle at rest. 
Now we construct 5-vectors from 4-vectors (10), (11). We suppose that a 5-vector

$$
\bar{p}=(m c, 0,0,0, m c)
$$

corresponds to a stationary particle of mass $m$.

The 5-vector of a particle, which moves with velocity $\vec{v}$, can be obtained by transformation to the moving coordinate system. Then the vector (15) takes the form

$$
\bar{p}_{m}=\left(\frac{m c}{\sqrt{1-\beta^{2}}}, \frac{m \vec{v}}{\sqrt{1-\beta^{2}}}, m c\right) .
$$

Similarly the 4-vector (11) transforms into 5-vector

$$
\bar{p}_{\omega}=\left(\frac{\hbar \omega}{c}, \frac{2 \pi \hbar}{\lambda} \vec{k}, 0\right)
$$

At the transition to a moving coordinate system the vector (17) does not change its form, only the frequency $\omega$ changes its value.

$$
\omega \rightarrow \omega^{\prime}=\frac{\omega}{\sqrt{1-\beta^{2}}} .
$$

Thus, in empty space in a stationary reference frame there are two fundamentally different object with zero and nonzero masses, which in the space of $G(1,4)$ correspond to the 5-vectors

$$
\bar{p}_{\omega}=\left(\frac{\hbar \omega}{c}, \frac{\hbar \omega}{c} \vec{k}, 0\right)
$$

and

$$
\bar{p}_{m}=(m c, 0,0,0, m c) .
$$

The vector (19) describes a photon with zero mass, the energy $\hbar \omega$, and the velocity $c$. The vector (20) describes a stationary particle of mass $m$. The photon has a momentum $p=\frac{\hbar \omega}{c}$, a massive particle has a momentum equal to zero. In the 5-dimensional space, these two vectors are isotropic, in Minkowski space only the vector (19) is isotropic.

The length of the 5-vector $\bar{l}=\left(x_{0}, x_{1}, x_{2}, x_{3}, x_{4}\right)$ is defined in accordance with the metric (+----) of the extended space $G(1,4)$. It equal to

$$
\bar{l}^{2}=x_{0}^{2}-x_{1}^{2}-x_{2}^{2}-x_{3}^{2}-x_{4}^{2} .
$$

It follows from the definition (21) that 5-vectors (16), (17) are isotropic vectors, ie their length is equal to zero.

$$
\bar{p}_{m}^{2}=\bar{p}_{\omega}^{2}=0 .
$$

If we restrict ourselves to Lorentz transformations in Minkowski space it is impossible to transform an isotropic vector into anisotropic one and vice versa. In other words in frame of the SRT photon can not acquires mass, and a massive particle can not be a photon. But in the Extended space $G(1,4)$ a photon and a massive particle can be related to each other by a simple rotation.

As it was already mentioned the parameter $n$ connects the speed of light in vacuum with that in the medium: $v=c / n$. Using it, one can parametrize the fifth coordinate in the $G(1,4)$ space. The value $n=1$ corresponds to the empty Minkowski space $M(1,3)$ in which light moves at the velocity $c$. The propagation of light in a medium with $n \neq 1$ is interpreted as an exit of a photon from the Minkowski space and its transition into another subspace of $G(1,4)$ space. This transition can be described as a rotation in the $G(1,4)$ space. All types of such rotations were studied in Ref. [1]. 
For hyperbolic rotation through the angle $\theta$ in the (TS) plane the photon 5-vector (19) with zero mass is transformed in the following manner (Ref. [1]):

$$
\left(\frac{\hbar \omega}{c}, \frac{\hbar \omega}{c} \vec{k}, 0\right) \Rightarrow\left(\frac{\hbar \omega}{c} \cosh \theta, \frac{\hbar \omega}{c} \vec{k}, \frac{\hbar \omega}{c} \sinh \theta\right)=\left(\frac{\hbar \omega}{c} n, \frac{\hbar \omega}{c} \vec{k}, \frac{\hbar \omega}{c} \sqrt{n^{2}-1}\right) .
$$

As a result of this transformation a particle with mass is appeared.

$$
m=\frac{\hbar \omega}{c^{2}} \sinh \theta=\frac{\hbar \omega}{c^{2}} \sqrt{n^{2}-1} .
$$

The velocity of this particle is defined by formula (7).

Under the same rotation the massive 5-vector (20) is transformed as

$$
\begin{aligned}
(m c, 0,0,0, m c) & \Rightarrow\left(m c e^{\theta_{ \pm}}, 0,0,0, m c e^{\theta_{ \pm}}\right) ; \\
e^{\theta_{ \pm}} & =n \pm \sqrt{n^{2}-1} .
\end{aligned}
$$

Under such rotation a massive particle changes its mass

$$
m \rightarrow m e^{\theta}, 0 \leq \theta<\infty
$$

and energy but conserves its momentum.

The rotation through the angle $\phi$ in the (XS) plane transforms the photon vector in accordance to the law

$$
\left(\frac{\hbar \omega}{c}, \frac{\hbar \omega}{c}, 0,0,0,\right) \Rightarrow\left(\frac{\hbar \omega}{c}, \frac{\hbar \omega}{c} \cos \phi, 0,0, \frac{\hbar \omega}{c} \sin \phi\right)=\left(\frac{\hbar \omega}{c}, \frac{\hbar \omega}{c n}, 0,0, \frac{\hbar \omega}{c n} \sqrt{n^{2}-1}\right) .
$$

Given this, the photon acquires the mass

$$
m=\frac{\hbar \omega}{c^{2}} \sin \phi=\frac{\hbar \omega}{c^{2} n},
$$

and velocity

$$
v=c \cos \phi=\frac{c}{n} .
$$

The vector of a massive particle is transformed in accordance to the law

$$
(m c, 0,0,0, m c) \rightarrow(m c,-m c \sin \phi, 0,0, m c \cos \phi)=\left(m c,-\frac{m c}{n} \sqrt{n^{2}-1}, 0,0, \frac{m c}{n}\right) .
$$

In this transformation the energy of a particle is conserved but its mass and momentum change

$$
\begin{aligned}
& m \rightarrow m \cos \phi=\frac{m}{n}, \\
& 0 \rightarrow-m c \sin \phi=-\frac{m c}{n} \sqrt{n^{2}-1} .
\end{aligned}
$$

It is easy to see that vectors (23), (25) and (27), (30) are isotropic.

It is important that photon mass, which is generated by transformations (23), (27), can have either positive and negative sign. This immediately follows from the symmetry properties of $G(1,4)$ space. As to the particles that initially had positive mass, after transformations (25), (30) it remains positive.

\section{The Mass of a System of Photons}

It is well known that one can compare a plain wave and vector (19) to a photon only in empty space. If there is some particle or field in the space in addition to initial photon it is necessary to describe this photon by other vector. In order to find this vector let's consider the system of two photons with a same energy. We suppose that these photons are moving in the same plane but in different directions. In the empty space these photons are described by 5 -vectors (19) 


$$
\left(\frac{\hbar \omega}{c}, \frac{\hbar \omega}{c^{2}} v_{x}, \frac{\hbar \omega}{c^{2}} v_{y}, 0,0\right)
$$

and

$$
\left(\frac{\hbar \omega}{c}, \frac{\hbar \omega}{c^{2}} v_{x},-\frac{\hbar \omega}{c^{2}} v_{y}, 0,0\right)
$$

Here $v_{x}, v_{y}, v_{z}$ are components of photon's velocity, they satisfy the condition $v_{x}^{2}+v_{y}^{2}+v_{z}^{2}=c^{2}$. In our case, $v_{z}=0$.

The energy $E$ of the system of two photons is $E=2 \hbar \omega$.

The momentum $P$ of the system of two photons is $P=2 \frac{\hbar \omega}{c^{2}} v_{x}$.

These photons do not interact with each other, therefor the system of two such photons is a free system and it must be described by an isotropic 5-vector. This vector reads

$$
\left(2 \frac{\hbar \omega}{c}, 2 \frac{\hbar \omega}{c^{2}} v_{x}, 0,0,2 \frac{\hbar \omega}{c^{2}} \sqrt{c^{2}-v_{x}^{2}}\right)=\left(2 \frac{\hbar \omega}{c}, 2 \frac{\hbar \omega}{c} \cos \alpha, 0,0,2 \frac{\hbar \omega}{c} \sin \alpha\right) .
$$

We see that one can associate with a system of two photons a mass

$$
m=2 \frac{\hbar \omega}{c^{2}} \sqrt{1-\frac{v_{x}^{2}}{c^{2}}}=2 \frac{\hbar \omega}{c^{2}} \sin \alpha .
$$

Here $\frac{v_{x}}{c}=\cos \alpha$ and $2 \alpha$ is an angle between directions of photons.

Let us compare now formulas (27) and (36). We see that in our case the angle $\phi$, which determines the rotation the (XS) plane, is equal to angle $\alpha$ that is a half of an angle between directions of photons.

The other approach to the problem of constructing a mass of a system of photons was proposed by Rivlin [12] [13] and Fedorov [14].

\section{Localization of Fields and Particles}

There is a natural way in the frame of ESM to establish a connection between mass of a particle and its size. It can be done with the help of an analogy between the dispersion relation for a free particle

$$
E^{2}=(c \vec{p})^{2}+m^{2} c^{4}
$$

and dispersion relation for a wave in the hollow metal waveguide

$$
\omega^{2}=\omega_{m n}^{2}+(c \xi)^{2}
$$

Here $\omega_{m n}$ is the critical frequency of the waveguide mode, and $\xi$ is a wave propagation constant.

The similarity of the ratios (37) and (38) drew the attention of many scientists. One can associated with the critical frequency $\omega_{m n}$ a parameter

$$
M=\frac{\hbar \omega_{m n}}{c^{2}} .
$$

This parameter has the unit of mass, and the question arises, if this quantity can be interpreted as a real mass? The mass, which acquires the electromagnetic field when it enters the waveguide. In the works of Rivlin, this problem was studied in a systematic way [12] [13]. Here we will not go into this problem, but we will mention only the fact that the mass $m$ is related to the width $a$ of a square waveguide by a relation

$$
a=\frac{\sqrt{2} \pi \hbar}{m c} .
$$

That is the value, which we propose to consider the characteristic linear parameter that is associated with the particle. 


$$
l=\frac{2 \pi \hbar}{m c} .
$$

The value (41) resembles the Compton wavelength of the electron, however, the physical meaning of it is very different. In the formula for Compton wavelength of the electron, the parameter $m$ is the rest mass of the electron, but in Equation (41) $m$ is the mass that a photon acquires when it is subjected by external influences.

In the empty space a free photon is described by a plane wave and has an infinite size. The mass of this photon is equal to zero, but its energy is finite. It is an idealized object. In does not exist in reality, because in reality there is no absolutely empty space. But when photon enters the space with external fields, it acquires a non-zero mass $m$. In accordance with the formula (40) a finite linear parameter l can be compared to this mass $m$. We consider the linear parameter $l$ as a size of a photon. Such reduction of an infinite format of a free photon to finite size of a photon in an external field us a result of action of this field.

In ESM an external action is described by rotations in Extended space $G(1,4)$. We have discussed above the rotations from the group $L(1,4)$, and set how changing the mass of the photon at these turns. Because a linear parameter $l$ expressed by the formula (41) using the mass of the photon, with its help it is possible to find the dependence of this parameter from the values define these rotations.

So, in the case of rotations in the plane (TS) dependence of the photon mass from the angle of rotation $\theta$ is determined by the formula (10). Substituting this expression into the formula (41), we obtain the expression for the parameter $l$ through the angle $\theta$.

$$
l=\frac{2 \pi c}{\omega \sinh \vartheta} .
$$

In the case of rotations in the plane (XS) dependence of the photon's mass is determined by the formula (27). With its help, we obtain an expression for the parameter $l$ through the angle $\psi$.

$$
l=\frac{2 \pi c}{\omega \sin \psi} .
$$

The rotation angles $\theta, \psi$ describe the value of external action. If this action tends to zero, the angles

$$
\theta, \psi \rightarrow 0
$$

the mass $m \rightarrow 0$ and the size of photon

$$
l \rightarrow \infty
$$

\section{Conclusion}

In given work, the generalization of Einstein's Special theory of relativity is proposed. It is the $(4+1)-$ dimensional Extended space model. It is shown that in the frame of this model, it is possible to compare the mass and size of the photon. In forthcoming works, we will discuss the problem of localization of massive particles.

\section{References}

[1] Tsipenyuk, D.Yu. and Andreev, V.A. (2000) Bulletin of the Lebedev Physics Institute, 6, 23. arXiv:gr-qc/0106093.

[2] Tsipenyuk, D.Yu. and Andreev, V.A. (2002) Bulletin of the Lebedev Physics Institute, 6, 1. arXiv:physics/0302006.

[3] Tsipenyuk, D.Yu. and Andreev, V.A. (2004) Bulletin of the Lebedev Physics Institute, 9, 10. arXiv:physics/0506002.

[4] Tsipenyuk, D.Yu. and Andreev, V.A. (2004) Bulletin of the Lebedev Physics Institute, 10, 13. arXiv:physics/0407144.

[5] Andreev, V.A. and Tsipenyuk, D.Yu. (2014) Natural Science, 6, 248-253. http://dx.doi.org/10.4236/ns.2014.64028

[6] Andreev, V.A. and Tsipenyuk, D.Yu. (2015) Tunneling of the Potential Barrier and Particle's Size in the Extended Space Model. Proceedings of International Meeting, Bauman Moscow State Technical University, Moscow, 29 June-2 July 2015, 20-32.

[7] Kobayashi, S. and Nomizu, K. (1963) Foundation of Differential Geometry, Vol. 1. Inderscience Publishers, New York, London, Sydney.

[8] Okun', L.B. (1989) Physics-Uspekhi, 32, 629. http://dx.doi.org/10.1070/PU1989v032n07ABEH002739 
[9] Okun', L.B., Selivanov, K.G. and Telegdi, V.L. (1999) Physics-Uspekhi, 42, 1141. http://dx.doi.org/10.1070/PU1999v042n10ABEH000597

[10] Okun', L.B. (2000) Physics-Uspekhi, 43, 1366. http://dx.doi.org/10.1070/PU2000v043n12ABEH000889

[11] Landau, L.D. and Lifshitz, E.M. (1994) The Classical Theory of Fields. World Scientific, Singapore.

[12] Rivlin, L.A. (1997) Physics-Uspekhi, 40, 291. http://dx.doi.org/10.1070/PU1997v040n03ABEH000215

[13] Rivlin, L.A. (1992) Soviet Journal of Quantum Electronics, 22, 771.

[14] Fedorov, M.V. and Vintskevich, S.V. (2016) Invariant Mass and Propagation Speed of Light Pulses in Vacuum. arXiv:1604.00227v3.

\section{Submit or recommend next manuscript to SCIRP and we will provide best service for you:}

Accepting pre-submission inquiries through Email, Facebook, LinkedIn, Twitter, etc.

A wide selection of journals (inclusive of 9 subjects, more than 200 journals)

Providing 24-hour high-quality service

User-friendly online submission system

Fair and swift peer-review system

Efficient typesetting and proofreading procedure

Display of the result of downloads and visits, as well as the number of cited articles

Maximum dissemination of your research work

Submit your manuscript at: http://papersubmission.scirp.org/ 